\title{
EMPIRICAL FINDINGS ON THE ASSOCIATION BETWEEN EDUCATION AND CHILD HEALTH STATUS: DISCUSSION
}

\author{
ROBIN BARLOW \\ Center for Research on Economic Development and Department of Economics, University of \\ Michigan, Ann Arbor, MI 48109, U.S.A.
}

All three papers in this section attempt to explain variations in child mortality. They do so with regression models in which a large number of independent variables appear in a reduced form obtained from an underlying structural system. We have numerous results to look at here, some of which are in line with common sense or original hypotheses, others of which are counter-intuitive.

There are a couple of the latter type that are worth noting, although they do not loom very large in these particular analyses. We normally think of the ownership of assets as being associated with better health conditions in the household. But there are at least two cases here where asset ownership is associated with worse health. In the paper by Simmons and Bernstein, it is large animals that cause the problem. One would think that ownership of large animals would denote greater wealth and a higher income, but it turns out that the animals increase the likelihood of tetanus and actually worsen infant mortality, particularly neonatal mortality. And in the case of Dr. Chowdhury's paper, boats are associated with worse health. The greater the number of boats, the worse off households are in terms of infant mortality. Dr. Chowdhury does not offer an explanation for this relationship in his paper, but in his oral presentation, he suggested that perhaps many of those households with large numbers of boats include fishermen, who would be among the poorest of the people being sampled.

These papers, which are essentially multivariate analyses of infant mortality using micro- or household data, manifest two general problems. To take care of these problems, we probably have to use other more detailed data sets. What I have to say here is not critical in the sense of saying that the authors could have done more with the data at their command. I think that they have done almost all that could possibly be done. But it would seem that there are some important questions still unanswered and, to answer those, one really needs new data sets. My remarks amount to suggestions for future research planning, rather than suggestions as to how the analyses presented in these papers could be improved. 
The two main problems are as follows. First, education is one of the independent variables in these analyses, and is of special interest to this conference. But education is closely associated with other possibly strong determinants of infant mortality and, in general, data are not available on these other determinants. Education in these regression analyses tends to represent the pure effect of education, and also the effect of the other socioeconomic variables with which it happens to be either statistically or causally related. Income is the major socioeconomic variable which is not represented explicitly in these regression analyses. Both for a scientific understanding of the mechanisms at work here, and for the sake of designing policy that might have an impact on infant mortality, it would be useful to be able to distinguish between the effect of education as such and the effect of income as such. To some degree the different papers try to grapple with this problem, but I do not think they solve it satisfactorily.

My second general area of concern is that none of the papers is able to throw much light on the precise mechanisms by which education might affect health conditions and mortality. Clearly there are a number of possible mechanisms. One is related to what I have just mentioned: that education is related to income, and that the effect of education on health perhaps occurs through income. Education leads to higher income and hence better nutrition, more expenditure on medical care, and so on, and that may be what improves health conditions. Or it may be that education of the mother leads to better nutritional planning within the family, income being held constant. Or it may be that standards of personal hygiene in the home are higher if the mother or father is more educated. Or it may be that education makes the parents more inclined to use western medicine.

Many different mechanisms are possible, and much of the attention of this conference is devoted to precisely this question. But the regression analyses cannot indicate which of these mechanisms is operating. Clearly, more detailed microdata are needed to solve that problem.

Related to this issue is the notion that the education variable used in these analyses is highly simplistic. Only two dimensions of education are available from the household data sets employed here. One is the number of years of schooling and the other is the distinction between primary and secondary schooling. But there are many other dimensions of education that conceivably could affect the relationship between education and health. It may be that curricula, or the subjects taught in the schools, are of some importance, and that education has more of an impact on health if the curriculum emphasizes scientific subjects as distinct from humanistic subjects. Clearly there are major differences in the curricula of schools in the Third World. Some schools, for example, emphasize religious subjects very heavily. In some Muslim countries in the past most formal education took 
place in Koranic schools, where the major item in the curriculum was rote learning of the Koran. The question arises, does this kind of schooling, as distinct from more secular types, have a different impact on health? Perhaps other dimensions of schooling, such as class size, the sex of the instructor, or the type of training that the instructor has received could conceivably have a differential impact on health conditions.

Some studies on the effects of education and various other variables in the United States have found that these other dimensions of education, besides educational attainment or the number of years of schooling, do have an impact. One study on the impact of education on future earnings showed that the quality of the school district, as measured by expenditures per pupil, did have an effect (a positive one, fortunately) on future earnings of children from that district. So it may be worthwhile in future research, if the data possibilities are there, to start distinguishing between different dimensions or aspects of education to see in which ways health is differentially affected.

The three papers recognize these two problems, and are not able to do much about them, given data limitations. Nonetheless they come up with several interesting findings. Let me turn to the three papers for some more specific comments.

The paper by Simmons and Bernstein reports as its main finding that post-neonatal mortality is determined to an important extent by preferences within the family for additional male or female children. These preferences or attitudes lead either to neglect or to extra care of new children, and hence have an important effect on mortality patterns. The education variable, relating to the education of the father and mother, is of somewhat less importance; it is not important at all at the neonatal stage, and at the post-neonatal stage is only of importance among female children. We see here the problem already alluded to: that education is representing not only itself, but also other variables with which it is statistically associated, particularly income. Simmons and Bernstein, in their discussion of this particular problem, do refer to some unpublished regressions in which they included both education and income simultaneously as independent variables. They report, without detailing the form of the equation or the values of the coefficients, that if the regression includes an income-type variable, which in their case is represented by landholdings, the result is that the education variable remains important, and the pseudo-income variable, landholdings, turns out to be unimportant. This result deserves to be reported in more detail. It would be noteworthy to discover that income is not a strong determinant of infant mortality, but that education is, even when the two determinants are considered simultaneously in the same equation. Or it may be that there are some specific problems with the landhold- 
ing variable that make it a poor proxy for income. There may be parallel problems with landholdings in India as there was with boats in Bangladesh.

The paper by Dr. Chowdhury reports that education is relatively unimportant as a determinant of mortality, a result which some people at this conference would find disappointing. He suggests that there may nonetheless be some indirect influence of education on health. This influence operates through an intermediate variable appearing in the analysis, the number of months of gestation. The length of gestation turns out to be a powerful determinant of neonatal and post-neonatal mortality in his analysis. It is possible, he suggests, that education lengthens gestation, and through this mechanism reduces infant mortality. There is indeed, and his argument rests on this, a positive simple correlation between education and length of gestation, although it seems to me rather weak, with a correlation coefficient of only 0.16 . Again, I think one would like to see a discussion of the precise mechanisms by which the education of the mother could be associated with the number of months of gestation. Does education operate indirectly through an income variable, so that in more highly educated families there is more income, and pregnant women have improved nutrition, which extends the period of gestation to term? Just what is the mechanism?

The formidable paper by Rosenzweig and Schultz has a tremendous wealth of findings that take a long time to digest. Several of them have been alluded to in the conference discussion and are certainly intriguing, like the results relating to temperature. The authors confront and are aware of the two problems that I mentioned: first, the question of whether education really represents itself or other things too, and secondly the general lack of insight on the detailed mechanisms through which education affects health conditions. Their failure to solve these problems does not, however, affect what is perhaps their main finding from the policy point of view. This is the finding that health care programs offered by the public sector, like free clinics and public hospitals, tend to be redistributive. The programs bring about the greater reductions in mortality among the less educated segments of the population. We should note that whether their education variable represents itself or income, the finding that the health programs are redistributive or egalitarian stands up either way. If education is standing for income, it is in the low-income groups where the programs are most effective. If education really represents itself, it is the uneducated groups which benefit most. Either way, the programs are obviously egalitarian in their impact on mortality. 